\title{
Magnetization and Losses for an Improved Architecture of Trapped-flux Superconducting Rotor
}

\author{
Vicente Climente-Alarcon, ${ }^{1}$ Anis Smara, ${ }^{1}$ Anup Patel, ${ }^{1}$ and Bartek A. Glowacki ${ }^{2}$ \\ University of Cambridge, Cambridge, CB3 OFS, UK \\ Algirdas Baskys ${ }^{3}$ \\ CERN, Meyrin, 1217, Switzerland \\ and \\ Thomas Reis ${ }^{4}$ \\ Oswald Elektromotoren GmbH, Miltenberg, 63897, Germany
}

\begin{abstract}
A hybrid electric configuration for aircraft propulsion yields several advantages, such as reducing the fuel consumption and take-off distance, improving control, and decreasing emissions. For such a scenario to occur, advances designed to increase the power-to-weight ratio of actual electric motors must be developed. Superconducting technologies offer the prospect of achieving such performances but at a cost of increased design and construction complexities. In that sense, stacks of high temperature superconductors have proven to trap high-current vortexes that provide a source of magnetic flux density for torque production without the need of current leads or other equipment in the rotor. However, these macroscopic currents must be induced prior to operation and remain undisturbed by variations in the magnetic flux density of the airgap, such as the ones caused by heating and demagnetization. This work presents the results of numerical computations on a new rotor architecture designed to facilitate the magnetization of stacks from a superconducting stator and prevent their demagnetization during torque production. The machine performance is assessed, and the expected survivability of the trapped-flux in stacks is compared to laboratory measurements.
\end{abstract}

\footnotetext{
${ }^{1}$ Research Associate, Department of Materials Science and Metallurgy.

${ }^{2}$ Professor, Department of Materials Science and Metallurgy, and also Professor of Energy at Institute of Power Engineering, Warsaw, Poland.

${ }^{3}$ Research Associate.

${ }^{4}$ Project Manager.
} 


\section{Nomenclature}

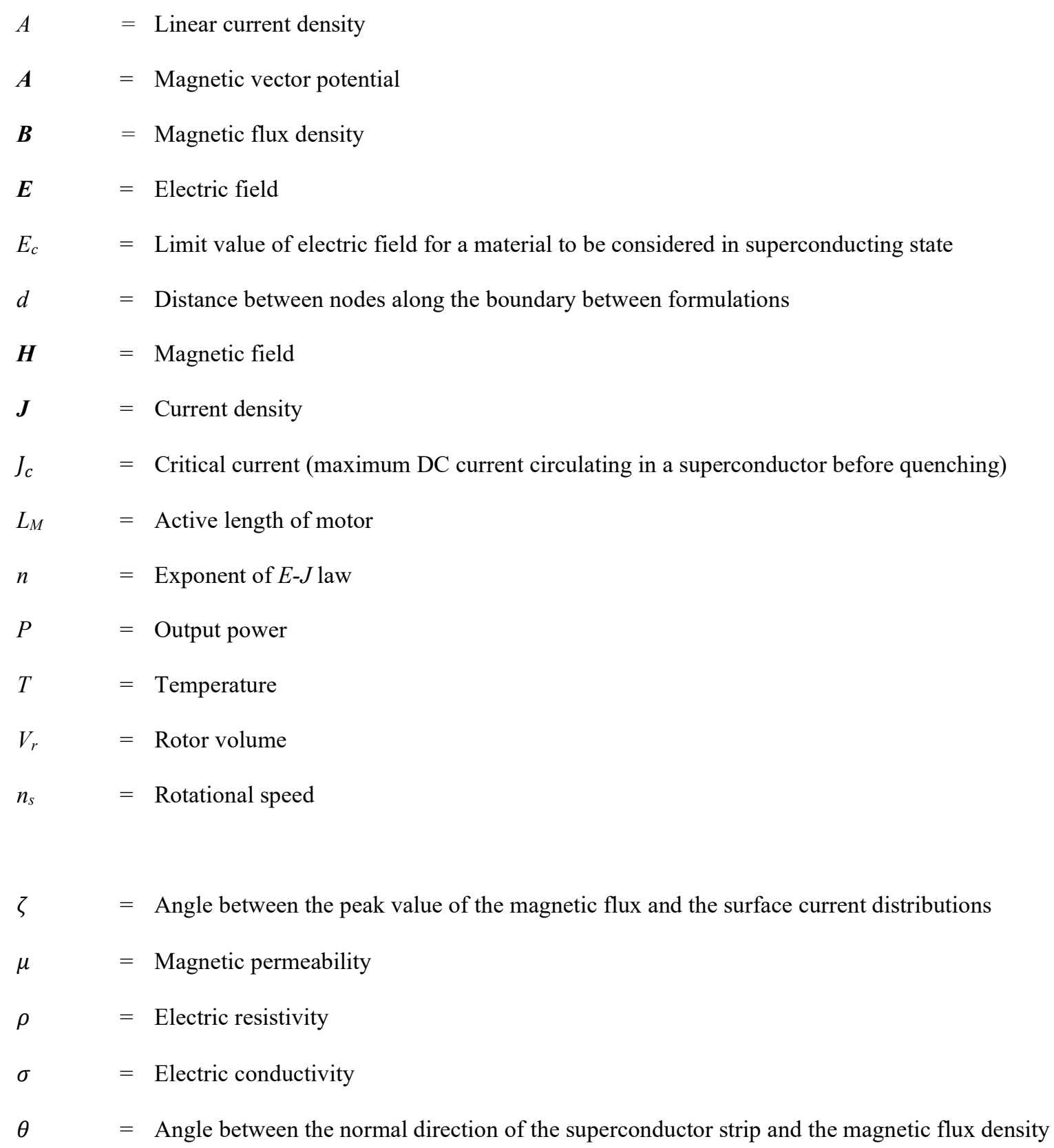

\section{Introduction}

Drevious analyses have shown that hybrid electric technologies will be able to meet the future demands of aircraft propulsion [1]. Under such architectures, the speed ratio between the fan and the core of a turbofan motor is bridged using a motor-generator arrangement equivalent to an electric transmission. Furthermore, once divided between propulsion and electricity generation, the corresponding elements can each be installed in the most aerodynamically favorable portions on the aircraft, allowing configurations that improve propulsion 
efficiency by ingesting the boundary layer, as proposed in [2]. Nevertheless, to make such a configuration feasible, the power density of the actual electric motor must be increased an order of magnitude from the actual state-of-the-art designs of $5 \mathrm{~kW} / \mathrm{kg}$ and up to $40 \mathrm{~kW} / \mathrm{kg}[3]$.

In the initial stages of designing an electric motor, the power density (proportional to the ratio $P / V_{r}$ where $P$ is the yielded power and $V_{r}$ is the volume of the rotor) is approximated as:

$$
\frac{P}{V_{r}}=n_{s} \hat{A} \hat{B} \cos \zeta
$$

where $\hat{A}$ is the peak value of the surface stator current, $\hat{B}$ is the peak value of the magnetic flux density - both considered sinusoidal-, $\zeta$ is the angle between both distributions, and $n_{s}$ is the rotational speed [4]. Some advances towards increased power densities have been achieved from larger $n_{s}$, that is, by repeating the same cycle but at a faster rate. However, the application of this machine in airliner propulsion requires the fan for a turbopropulsor to move at a slow speed, which would exclude this approach unless a gearbox, with its associated weight, is added. Similar to wind generation, the trend has been to eliminate this element [5], thus, for airliner propulsion a low speed/very high torque electric motor is required. This is a highly demanding design that can be achieved either by acting on the currents of the stator $\hat{A}$ or the flux produced by the rotor $\hat{B}$ or both given the sharp increase in performance that is needed.

Superconductive technology may be able to accomplish the desired increases in both $\hat{A}$ and $\hat{B}$ [6]. After decades of manufacturing improvements, high temperature superconductor materials can carry hundreds of amperes across cross sections below $0.1 \mathrm{~mm}^{2}$, even in the presence of an external magnetic flux. The use of a high-temperature superconductor in the stator winding of an electric machine would multiply the current capacity, which acts on $\hat{A}$ in Eq. (1). A similar arrangement was attempted relatively early for rotors in synchronous machines [7], as the electromagnetic conditions for that part of the machine are more benign. This increases $\hat{B}$ in Eq. (1). However, a superconducting rotor is an element that, from a mechanical perspective, must withstand a noticeable shrinkage during cooling and, from a thermal perspective, must feature an internal circuit for refrigeration down to $10-20 \mathrm{~K}$ along with its corresponding thermal insulating couplings. A superconducting winding adds a secondary electric circuit with coils and feeders, which increases the complexity of the rotor and casts doubt on the ability to implement this approach in this field.

As a result, the authors proposed developing a fully-superconducting motor demonstrator for aircraft propulsion applications that simplifies the rotor construction by eliminating the use of current leads or other feeds to the rotor field winding. This is accomplished by utilizing an arrangement similar to that employed in 
permanent magnet motors. In this case, an assembly of high temperature superconducting tapes are cut and piled together. After being magnetized, the superconducting layers maintain a macroscopic current flow in the form of a vortex so long as refrigeration is provided and there are no variations of the magnetic flux density (Fig. 1). This solution is structurally sound as the tape exhibits the mechanical properties of the substrate on which the superconductor is deposited, which is usually a superalloy. Unlike bulk materials, the proposed design can be easily manufactured and unlike coils, point defects are not a problem, since they are smoothed out by the other layers. It is even possible to bend the design and adapt it to different shapes [8]. Such characteristics enabled a record $17.7 \mathrm{~T}$ being trapped using the hybrid configuration of such a stack [9].

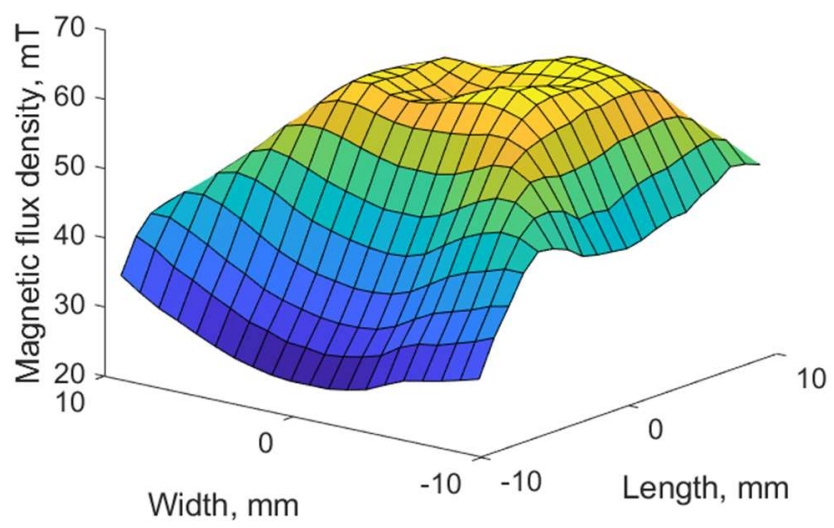

Fig. 1 Magnetic flux density trapped at the center of two layers of AMSC tape $(46 \times 46 \mathrm{~mm})$ at a temperature of $77 \mathrm{~K}$.

Nevertheless, two important problems arise from the application of trapped-field magnets as the source of the magnetic flux density in an electric machine. First, the supercurrents must be induced prior to operation. Second, any magnetic flux variation will induce an electric field that interacts with the macroscopic currents within the stack, yielding heat losses if the flux is normal to its surface [10] or demagnetization if the flux is parallel (cross-field) [11]. These variations are inherent to the airgap of an electric machine due to the discrete distribution of the stator coils and slots.

Two approaches are followed in the industry to avoid the influence of these harmonics in conventional rotors, which cause heating and weakening of the remanent flux when acting on permanent magnets: covering the rotor with a conductive sleeve to insulate its interior from high frequency components or burying the magnets inside the rotor iron where the magnetic environment is composed almost entirely of the constant (DC) component. However, for a fully superconducting machine, the first solution generates additional heat that must be removed by the rotor cooling system at extreme cryogenic temperatures [12]. The second solution greatly hinders the magnetization of buried (interior mounted) superconducting stacks from the superconducting stator 
since the rotor iron adds leakage paths around the stacks that remove much of the flux and weakens it to lower levels $[4,13]$. In addition, pulsing is in principle not possible in this case.

These conflicting requirements were previously identified [3] and addressed through the solution devised and demonstrated in this work, which is presented with the assistance of a 2D finite element model that weakly couples two formulations [14]. The model allows a superconducting machine to be treated roughly as a conventional one during this stage of its development and yields numerical results for both its performance (torque) and the expected losses in the stacks. It also provides an insight about their demagnetization rate through the value of cross-field that affects the stacks, and, therefore, about their operation span. Losses and cross-fields are compared to early assumptions and some laboratory measurements to assess the applicability of the proposed design. For this purpose, the remainder of this work is organized as follows: Section III introduces the main architecture of the proposed motor, including the novel rotor lay-out; Section IV summarizes the finite element formulation used to simulate the superconducting stacks during magnetization and operation inside the machine, already introduced in [14]; Section V yields the results in two points (magnetization and operation); and Section VI presents the conclusions.

\section{Motor Architecture}

The final configuration of the fully superconductive demonstrator features the classic radial electromagnetic construction with a distributed winding, a double cryostat thermal configuration [15] with independent cooling for the stator and rotor, and a toothless mechanical construction where the stator windings must be supported with a non-magnetic structure, which is not depicted in the simulations. Further mechanical aspects of this configuration represent a sharp departure from conventional machines to accommodate the nearly $300 \mathrm{~K}$ temperature reduction needed for operation.

Stacks of superconductive tapes are used as the permanent magnets. American Superconductor (AMSC) rare-earth barium-copper oxide (REBCO) tape manufactured to be $46 \mathrm{~mm}$ wide (used to achieve the record trapped field [9]) provides good characteristics for this application due to the uniformity of the trapped flux (shown in Fig. 1) and its relatively high critical current density. This simple arrangement of the magnetic flux sources simplifies the construction of the rotor compared with other approaches that require current leads [5], since the rotor is an element of great complexity due to the required helium cooling system circulating between an internal fixed insert and the external moving sleeve [15]. The moving sleeve, which provides the mechanical attachment using dovetails for the electromagnetic-active component of the rotor, is maintained at $25 \mathrm{~K}$ during operation. The characteristics of the simulated motor are presented in Table 1. 
Table 1 Motor characteristics

\begin{tabular}{lc|lc}
\hline \hline Stator external diameter & $236 \mathrm{~mm}$ & Number of phases & 3 \\
Stator iron internal diameter & $214 \mathrm{~mm}$ & Number of pole pairs & 5 \\
Stator winding distance & to & & \\
center & $101.5 \mathrm{~mm}$ & Number of coil sides per pole and phase & 1 \\
Stator coil cross section & $4 \times 10 \mathrm{~mm}$ & Stator turns per coil & 7 \\
Stack thickness & $5 \mathrm{~mm}$ & Supply frequency & $500 \mathrm{~Hz}$ \\
Rotor yoke external diameter & $190 \mathrm{~mm}$ & Operating stator current (RMS) & $400 \mathrm{~A}$ \\
Rotor yoke internal diameter & $150 \mathrm{~mm}$ & Magnetizing stator current (DC) & $1,600 \mathrm{~A}$ \\
Machine length & $175 \mathrm{~mm}$ & Torque & $500 \mathrm{~N} \cdot \mathrm{m}$ \\
\hline \hline
\end{tabular}

As for the stator, the solution devised for the rotor departs from existing ones. We initially considered the common configurations of a surface mounted (Fig. 2a) and internally-mounted stacks (Fig. 2b). The results of the conventional 2D finite element (FE) computations for a similar magnetization and operating conditions expected for the demonstrator are shown in Table 2. Clearly, a higher peak magnetic flux density and flux per pole can be obtained with surface mounted stacks. However, the normal and tangential variations of the magnetic flux density during operation are relatively large, despite the suppression of teeth in the stator, with losses rising as the third power of the field variations [10]. Interior mounted stacks are particularly well protected against tangential variations (cross-fields) that cause demagnetization, with values an order of magnitude below the surface mounted ones, however, their magnetization under the simulated conditions is poor, with a peak remanent flux and flux linked to the stator being half or less than the first configuration due to the presence of the iron bridges that are necessary to fix the pole section in place.

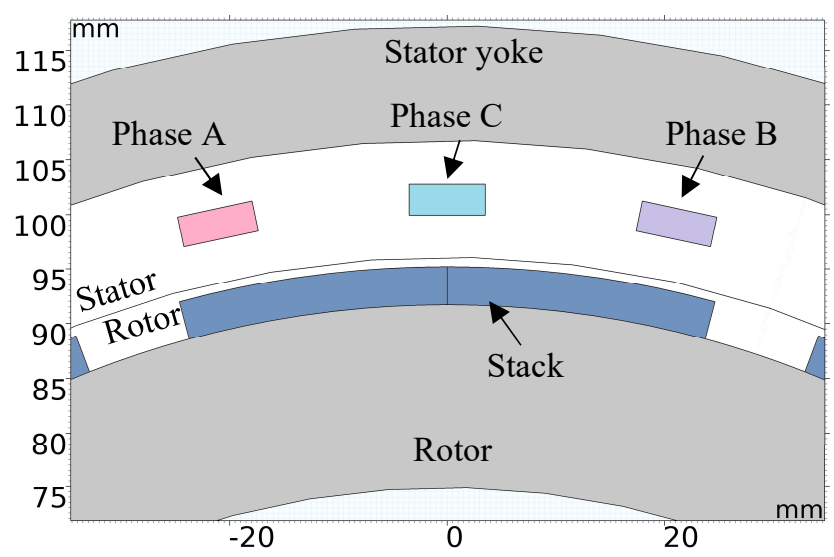

a) 


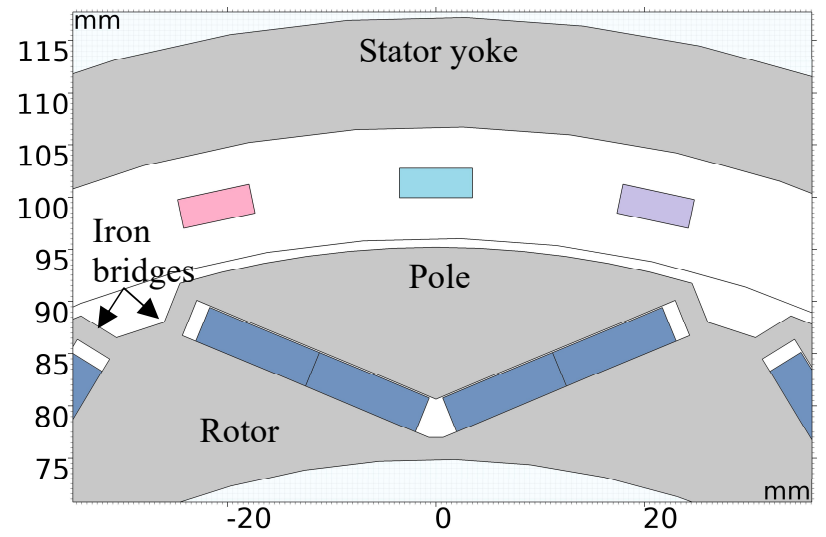

b)

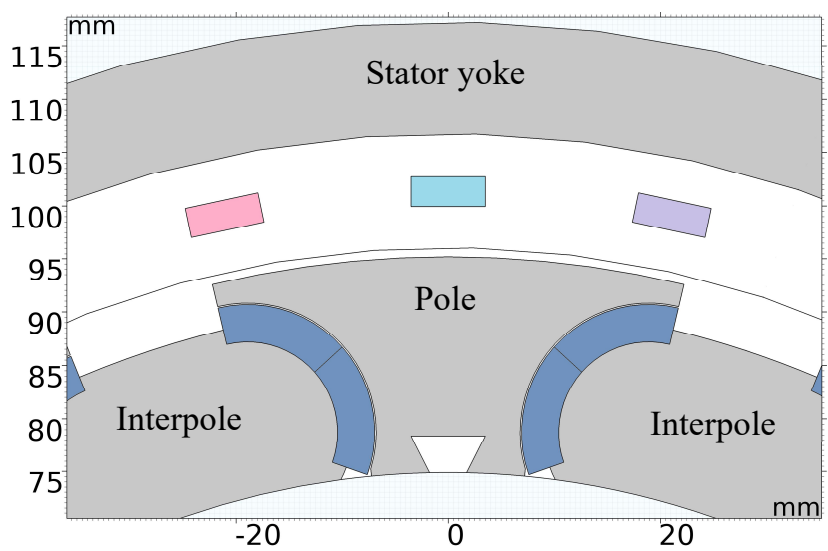

c)

Fig. 2 Geometries considered for the demonstrator: surface mounted stacks (a), interior mounted (b), and C-shaped (c).

Table 2 Magnetic characteristics of the stacks in each geometry

\begin{tabular}{cccccc}
\hline \hline Stack geometry & $\hat{B}_{\text {rem }}(\mathrm{T})$ & $\begin{array}{c}\text { Flux per } \\
\text { pole }(\mathrm{Wb})\end{array}$ & $\begin{array}{c}\max \left(\Delta B_{\text {norm }}\right) \\
(\mathrm{mT})\end{array}$ & $\begin{array}{c}\max \left(\Delta B_{\text {tan }}\right) \\
(\mathrm{mT})\end{array}$ & $\begin{array}{c}\text { Stack length } \\
(\mathrm{mm})\end{array}$ \\
\hline Surface mounted & 1.35 & $1 \cdot 10^{-2}$ & 100 & 150 & 49.8 \\
Interior mounted & 0.67 & $4.6 \cdot 10^{-3}$ & 25 & 20 & $2 \times 22.8$ \\
C-shape & 0.91 & $6.9 \cdot 10^{-3}$ & 45 & 40 & $2 \times 25.2$ \\
\hline \hline
\end{tabular}

These apparently incompatible requirements of surrounding the stacks with iron to protect them against the remaining airgap harmonics while avoiding leakage paths prompted the development of the C-shape configuration (Fig. 2c). Unlike permanent magnets, the high resistance at compression in the normal direction provided by the substrate allows using the stacks as structural elements. This enables the removal of iron bridges that are responsible for most of the leakage in conventional configurations. In this case, the interpole sections are locked in place by the stacks themselves and their load is transmitted to the poles, which protect the stacks from variations in the magnetic flux density during operation, favors magnetization through a low reluctance 
path along the $d$-axis direction (the center of the pole) and conveys the mechanical loads to the internal part of the rotor, which is also responsible for cooling, using a dovetail attachment. Furthermore, the length of the stacks is maximized from the curved shape that defines its name (C-shape). This profile also allows for a larger pole shoe pitch, which increases the saliency and shielding for the stacks while providing the necessary clearance for the dovetails at their lower ends.

In this configuration the values of the magnetic flux variation are roughly twice those from the buried stacks. However, they are still much smaller than surface mounted stacks. While the proposed configuration improves the remanent flux levels, they are still lower than conventional permanent magnets. Better magnetization methods must be developed in the future for this solution to be competitive.

In previous studies and the remainder of this work, a field cooling magnetization has been assumed. This procedure has a straightforward application to fully-superconducting motors with a double cryostat and distributed winding by first cooling the stator. Then , a very high DC current is then circulated along the $d$-axis of the now superconducting winding before finally cooling the rotor. When the DC current in the stator is slowly decreased, currents are induced in the rotor stacks that oppose the reduction of the flux. Zero field cooling involves applying at least twice the intended trapped flux, which requires more demanding conditions [16]. One variant of this later procedure is pulse magnetization, which is especially widespread in laboratory environments to study samples and some preliminary research advises its use in motors [17]. However, its actual implementation in an electric machine remains problematic. Superconducting windings cannot be pulsed as they suffer from quenching when the critical current density is reached. To overcome this, specially-designed coils must be installed in the stator of a fully superconducting machine. Furthermore, reproducibility of the flux levels attained in one stack cannot be guaranteed since pulsing creates complicated current patterns inside the superconductor [8]. In this project, the field cooling option was chosen as a simpler solution for such small machine. This allows magnetizing all the poles simultaneously and trapping uniform values of the magnetic flux density in the stacks [18].

\section{Finite Element Model}

Traditional radial electric machine design procedures determine its electromagnetic state only in the crosssection. This greatly simplifies Maxwell's equations, since for 2D applications and for the low frequencies in which they operate only a scalar state variable needs to be solved: the component of the magnetic vector potential $\boldsymbol{A}$ perpendicular to the plane, $A_{z}$, which is obtained from: 


$$
-\nabla \cdot\left(\frac{1}{\mu} \nabla A_{z}\right)+\sigma \frac{\partial A_{z}}{\partial t}=J_{z}
$$

where $\mu$ is the magnetic permeability, $\sigma$ the conductivity of the material, and $J_{z}$ the current density parallel to $A_{z}$. The Coulomb gauge $\nabla \cdot \boldsymbol{A}=0$ is used to fully determine $\boldsymbol{A}$, from where we obtain the magnetic flux density $\boldsymbol{B}$ simply as its curl:

$$
\boldsymbol{B}=\nabla \times \boldsymbol{A}
$$

During the design of an electric machine, the $A$-formulation is completed by further numerical techniques that permit, for instance, accounting for the relative movements of the rotor and stator, the computation of the yielded torque and also the influence of 3D effects by using lumped parameters introduced in coupled current and voltage circuit equations. Overall, computer codes based in the $A$-formulation provide the necessary answers to develop a standard conventional electric machine [19].

This well-established procedure cannot be hastily extended to study devices with superconductive regions. Type II superconductors can carry more current since microscopic conventional areas exist within them to provide a path for the magnetic flux lines to cross an otherwise diamagnetic material. The oscillations in these flux lines, such as from thermal activation, induce an electric field whose interactions with the circulating current creates a dissipative effect [20]. This is accounted for using a finite and highly non-linear resistivity in the form [14]:

$$
\rho(|J|)=\frac{E_{c}}{J_{c}}\left(\frac{|J|}{J_{c}}\right)^{n-1}
$$

with for the most general case the dependences $J_{c}=J_{c}(\boldsymbol{B}(\theta), T)$ and $n=n(|\boldsymbol{B}|)$. These values for the critical current $J_{c}$ are obtained from detailed material characterizations at different temperatures $T$, magnetic flux densities $\boldsymbol{B}$, and flux angles $\theta$. The $\boldsymbol{J}$ accounts for the current density at the point where the resistivity is computed, while the threshold field $E_{c}$ is usually taken as $1 \mu \mathrm{V} / \mathrm{m}$. The evolution of the exponent $n$ is more difficult to parametrize and usually requires averaging several measurements under different conditions.

The insertion of the inverse of Eq. (4) (with the electric field as input variable) into Eq. (2) as the conductivity $\sigma$ to assess the current distribution in superconducting materials creates numerical convergence problems since this variable is unbounded at the origin (for the initialization value $|\boldsymbol{E}|=0$, the conductivity $\sigma$ increases towards infinity) [14]. Some mitigation methods have yielded results by modeling superconductors 
using the $A$-formulation, but a more rigorous technique consists of employing the $H$-formulation instead. This approach is derived from Faraday's and Ampere's laws in the form:

$$
\frac{\partial \mu \boldsymbol{H}}{\partial t}+\nabla \times \rho(\nabla \times \boldsymbol{H})=0
$$

The direct use of Eq. (4) in Eq. (5) to obtain the current distribution in a superconductor provides a slow increase of $\rho$ for small values of $\boldsymbol{J}=\left[J_{z}\right]$, such as the ones in the superconductor near the beginning of the simulation. On the other hand, a vector quantity must be solved as $\boldsymbol{H}=\left[H_{x} H_{y}\right]^{T}$. Nevertheless, the addition of this formulation into the commercial finite element software COMSOL has popularized its use among the superconductor community. For example, this approach was employed to design a hybrid stack that trapped the actual record magnetic flux density of $17.7 \mathrm{~T}$ [9].

The development of a solution that combines both forms to solve the Maxwell's equations would benefit the study of different electric machine topologies. This would provide easier modeling and processing of the superconducting regions while allowing the utilization of the classic electromechanical principles to check the design performance. In this case, differences between the superconducting and conventional motors at this phase of their development would effectively be erased. Grilli et al. proposed and implemented a weak coupling of both formulations using a tramodel boundary in COMSOL [14]. This approach was applied here to simulate the behavior of the novel rotor lay-out during magnetization and operation. The inter-formulation boundary is set in the airgap of the machine, within the rotor region, next to and parallel to the moving boundary between the stator and the rotor. In this way, the stator is modeled conventionally (but with a much higher current density in its winding) using the $A$-formulation. Whereas the rotor, iron, stacks, and a part of the airgap utilize the $H$ formulation. The relative movement between the rotor and stator meshes is coupled in the $A$-formulation as is done for conventional machine simulations, which is an easier procedure due to its scalar nodal values.

The materials for the stator and rotor yokes are assumed to be silicon steel M270-35A, whose behavior at cryogenic temperatures is better understood compared with Fe-Co alloys and has higher magnetic saturation values. The superconducting regions are considered as filled with homogenized AMSC tape, which features a critical current of $391 \mathrm{~A} / \mathrm{cm}$-width at $77 \mathrm{~K}$ with a self-field and an engineering current density of $4.49 \cdot 10^{8} \mathrm{~A} / \mathrm{m}^{2}$. The substrate on which the superconductor is deposited is a Ni-5at\%W alloy with a yield stress of $257 \mathrm{MPa}$ [9]. The tape has a width of $46 \mathrm{~mm}$ and a thickness of $87 \mu \mathrm{m}$, which means that a single tape immersed in liquid nitrogen can carry up to $1.8 \mathrm{kA}$ before quenching. This transport current value more than doubles at liquid hydrogen temperatures. 
Consistent with the operation of the demonstrator, two stages are simulated using the combined formulation: an initialization that consists of field cooling magnetization of the stacks and the subsequent, motoring with loss computation. The variations in the magnetic flux density on the stacks during operation are also utilized to qualitatively assess their survivability against demagnetization by comparing the values of the cross-field obtained with their effects in experimental measurements. As mentioned, the field cooling process consists of applying a magnetic flux density on the stacks prior to reducing their temperature below the critical value (Fig. 3). DC currents in the stator supplied along the $d$-axis are used for this purpose. When these currents are slowly ramped down (Stage 3 of Fig. 3), currents are induced in the superconducting layers inside the stacks, which effectively traps the flux level and reproduces the applied value [21].

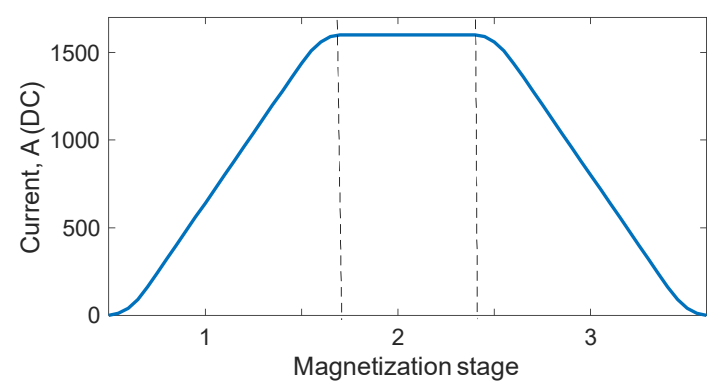

a)

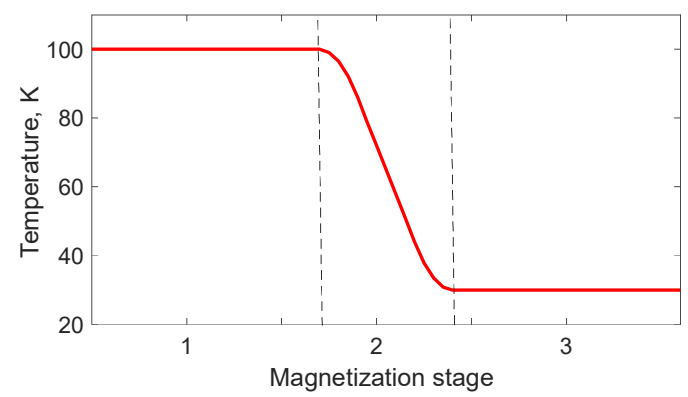

b)

Fig. 3 DC current profile fed to the stator (a) and rotor temperature (b) during the field cooling magnetization process.

The commercial finite element software COMSOL, which is widely known in the scientific community, is employed to obtain the results presented in this work [22]. The novelty applied here is utilizing the approach of [14], which establishes a common boundary between the $H$ - and $A$-formulations along which the state variables are weakly coupled. This means that a discretization error between the formulations at each side of the interface is allowed. The values of this error for the normal component of the common quantity magnetic flux density $\boldsymbol{B}$ are depicted in Fig. 4 as function of the distance $d$ between nodes along the boundary. As shown, the error 
decreases as the mesh is refined following a first order slope; however, the computational time doubles during that same span.

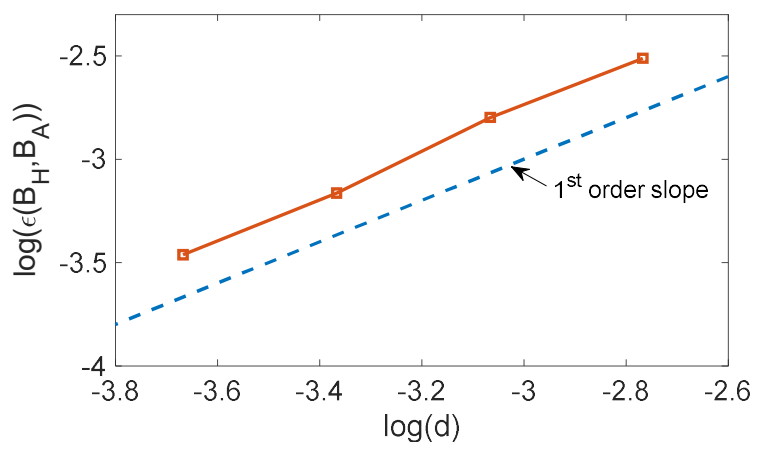

Fig. $4 \mathrm{L1}$ norm of the coupling error between formulations as a function of $d$.

Further sources of inaccuracy that are not shared with the computation of conventional electric machines stem from the homogenization procedure conducted in the stacks and variations in the material characteristics at deep cryogenic temperatures $(25-30 \mathrm{~K})$. Stacks are evidently composed of more than 50 separate layers, which are not singled out in the FE model, where the averaged properties of the superconductor plus the substrate are considered. In addition, variations in the material properties at these temperatures begin to depart from the trends observed at higher ones [5]. However, due to the ferromagnetic materials surrounding them, the stacks are expected to work in a similar way as in [9] with a magnetic flux during magnetization and operation primarily in the normal direction and under a similar range of temperatures.

After magnetization, the AC currents in the stator are ramped up and the rotor begins to turn when the maximum torque is reached (Fig. 5). The load angle for the maximum torque is determined from the timeharmonic simulations on a conventional model of the same motor geometry.

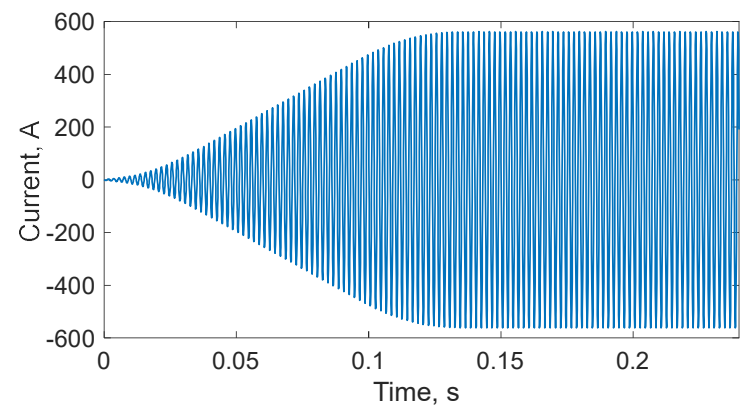

Fig. 5 Current fed in phase A during motoring simulation. 


\section{Results}

This section is divided in two subsections. The first discusses the results of the magnetization process and the second focuses on the output of the motoring simulation. In this later, the applicability of the proposed design is examined in terms of the output torque, electromagnetic losses in the stacks, and cross-field level (which leads to demagnetization), while operating the machine.

\section{A. Magnetization}

The simulation results for the field cooling magnetization process on the motor architecture proposed in this work are shown in Fig. 6. The first diagram (Fig. 6a) complements Fig. 3 as it illustrates the rising current for a single stack, which is computed as half the value of the integral of $\left|J_{z}\right|$ over its cross section. The maximum value is $5.4 \mathrm{kA}$ with a standard deviation between stacks of $22 \mathrm{~A}$. This small difference is attributed to the numerical approach. The modest value for the induced currents is motivated by the magnetization procedure and the large airgap resulting from the suppression of the stator teeth. Therefore, the stack is greatly undersaturated, which, as shown in Fig. 6b, yields a trapezoidal shape for the magnetic flux density at its pole-facing surface with heavy leakage at both sides due to the rapidly descending currents at its edges. The value of the magnetic flux density at the middle of the stack is only $0.75 \mathrm{~T}$ while the average only reaches $0.64 \mathrm{~T}$, which is well below what is achievable from conventional permanent magnets. The necessity of adding a clearance of $0.25 \mathrm{~mm}$ consisting of a non-ferromagnetic material around the stack for computational reasons accounts for the differences in the values expected from Table 2, which are based on conventional simulations with imposedcurrent density regions. Nevertheless, the flux per pole is calculated to be $7 \cdot 10^{-3} \mathrm{~Wb}$, due to the distinct current profile in the superconducting regions compared with that used to populate Table 2 (trapezoidal vs. triangular) and from the different saturation patterns these profiles create in the pole. The flux values can be increased further using, for example, additional coils in the stator for magnetization purposes. This is because the maximum average trapped magnetic flux density for $5-\mathrm{mm}$ stacks is $2 \mathrm{~T}$, which is well above the $1.33 \mathrm{~T}$ provided by state-of-the-art permanent magnets. Nevertheless, this supplementary winding cannot be arranged in the small machine designed here. 


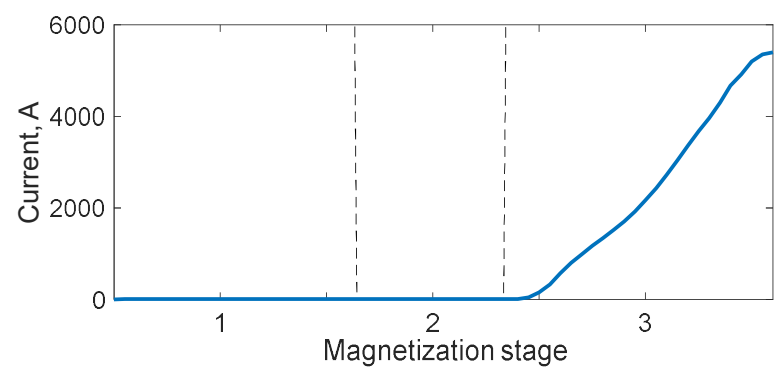

a)

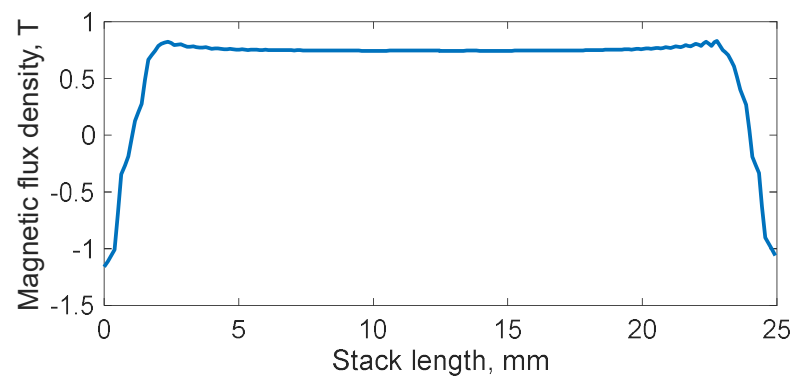

b)

Fig. 6 Current in the stack during the magnetization process (a) and the achieved magnetic flux density profile (b).

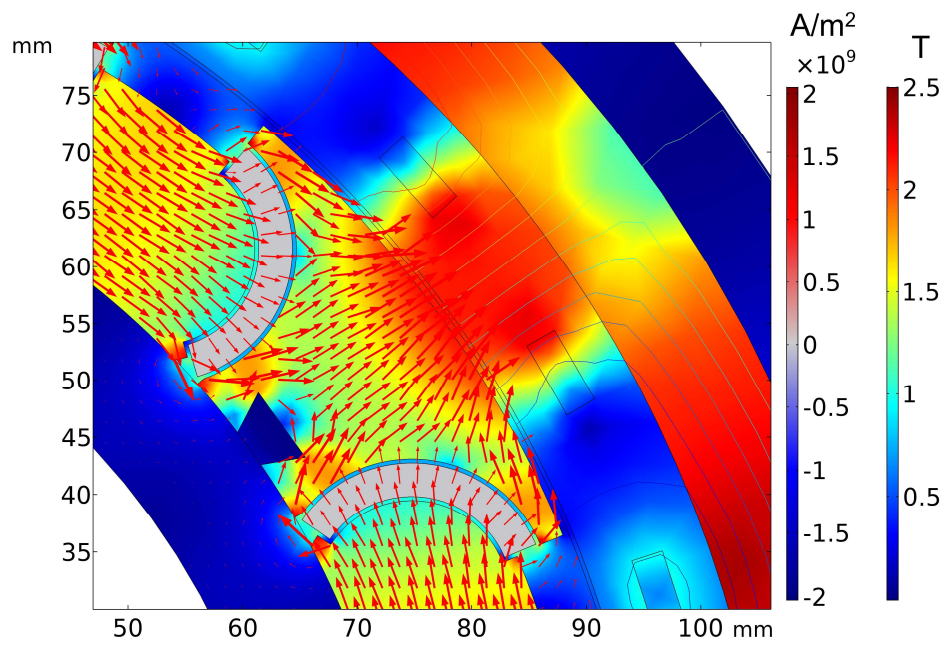

a) 


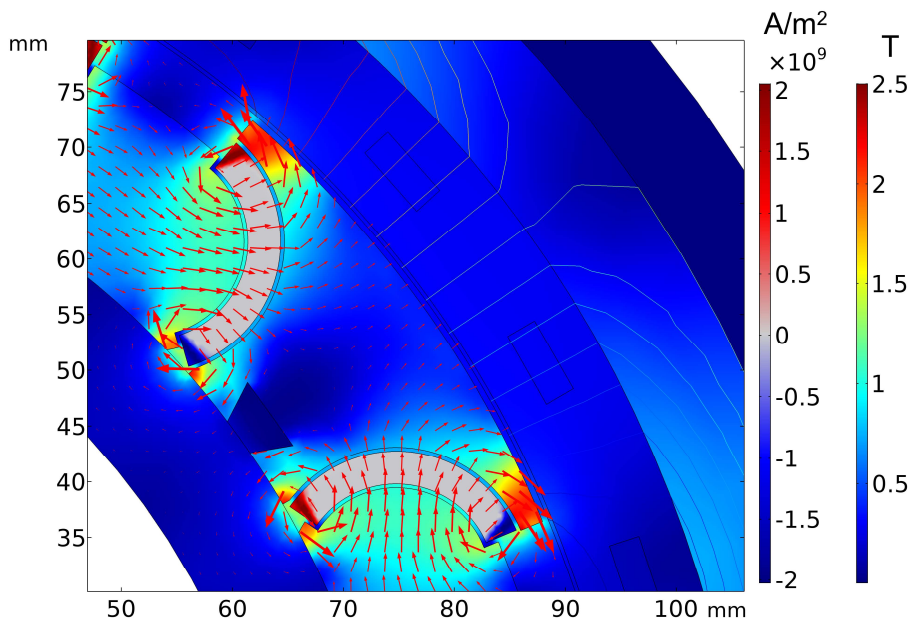

b)

Fig. 7 Magnetic flux density and current density in the stacks at the middle (a) and end (b) of the magnetization. The arrows indicate the magnitude and direction of the magnetic flux in the area of the $H$ formulation.

Figures $7 \mathrm{a}$ and $\mathrm{b}$ present the distributions of the current density in the stack and the magnetic flux density in the remainder of the machine cross section at the middle ("Stage 2" in Figs. 3 and 6a) and at the end of the magnetization procedure, respectively. The arrows have the same scale and represent the magnetic flux magnitude and direction in the $H$-formulation area of the cross-section. The effects of feeding the three phases with a 1,600 A DC is illustrated in Fig. 7a. In this case, there is a heavy magnetic saturation in the iron and no current in the stacks yet, since the temperature is above the critical value $T_{c}$ for the superconductor. In Fig. $7 \mathrm{~b}$, the limited loading of the stacks is clearly depicted from the induced currents flowing along its edges (both inwards and outwards) and perpendicular to the cross section while the center remains empty. Leakage at the edges is limited by the iron saturation at the pole tips and its bottom. In any case, this effect is very significant since it must be accounted for twice: during the magnetization the magnetic flux induced by the stator coils skips the stacks, and in operation the large airgap in the design implies the flux inverts its sense in these areas. This creates a significant return flow, but one that is always smaller compared with what would happen using the traditional configuration of interior-mounted permanent magnets. Counterintuitively, thinner stacks do not improve the machine performance because a greater saturation induces a more triangular magnetic flux density profile in the stacks, which exacerbates this fringe leakage.

\section{B. Motoring}

Similar to conventional machine studies in which a time-harmonic simulation is used to obtain the initial values, this work initialized the motoring simulation using the results of the last time-step obtained from the 
previous magnetization process. This reproduces the actual operational procedure to be followed in the demonstrator. The magnetic flux distribution in the cross-section of the machine after magnetization is shown in Fig. 8a and during operation in Fig. 8b. The effects of the stator currents are clearly appreciated as they heavily saturate the forward tip of the pole and increase the leakage from the interpole region. Figure $8 \mathrm{c}$, which corresponds to Fig. $7 \mathrm{~b}$ under motoring conditions, clearly illustrates the distortions due to saturation of the currents flowing in the stack below the pole tip.

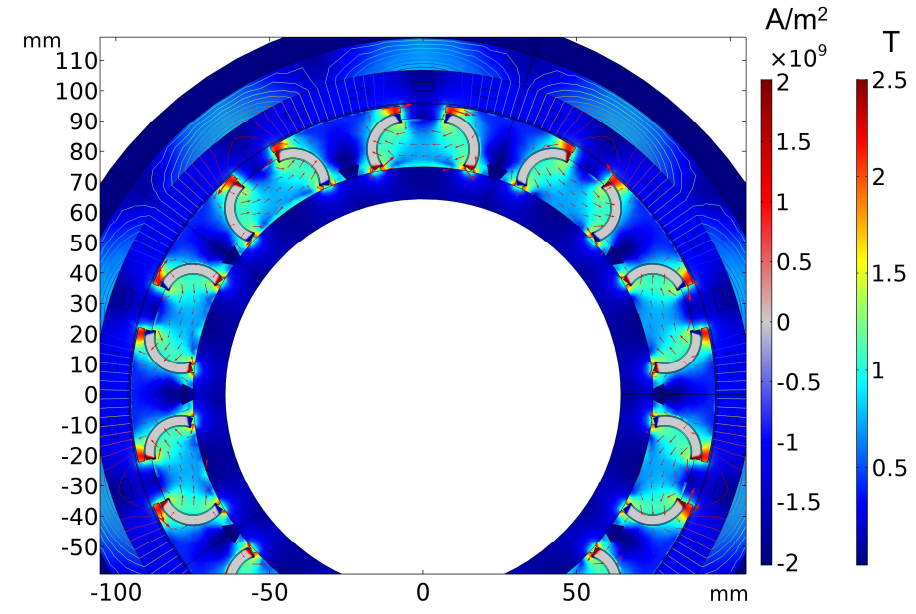

a)

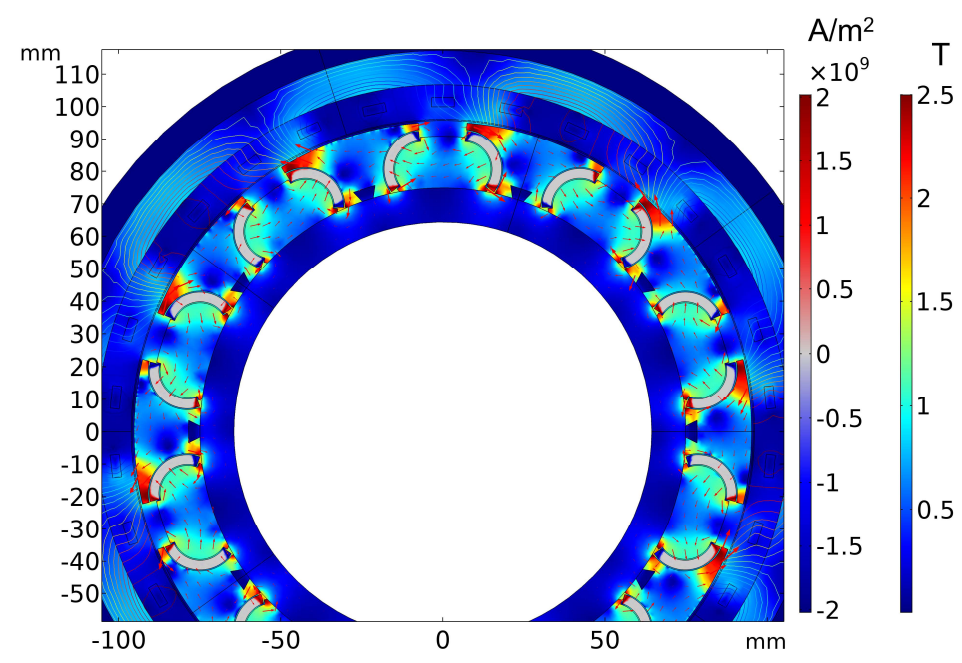

b) 


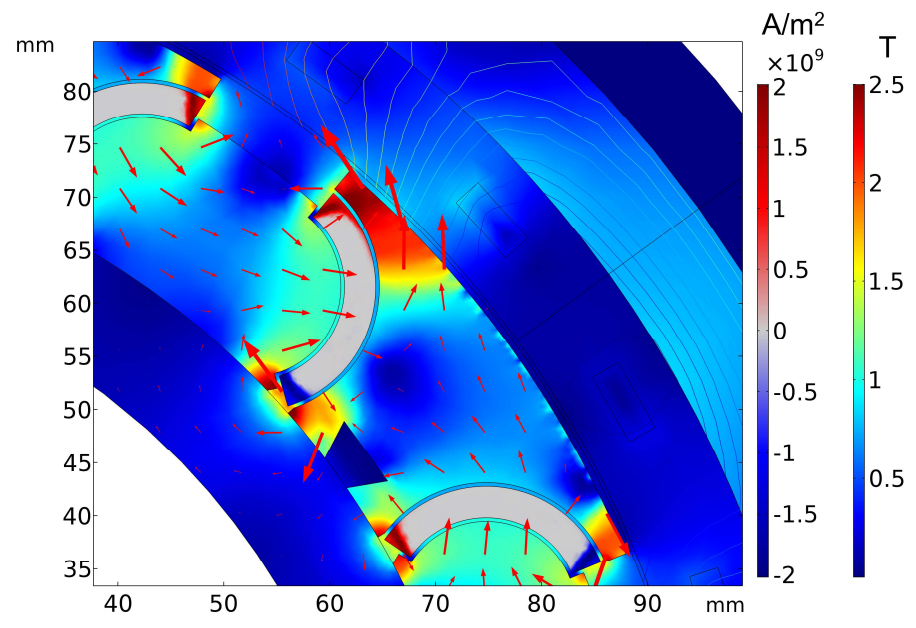

c)

Fig. 8 Magnetic flux density and current density in the stacks after magnetization (a), and during operation (b) and (c). The arrows indicate the magnitude and direction of the magnetic flux in the area of the $\boldsymbol{H}$-formulation

\section{Torque}

The profile for the average torque during the simulation is shown seen in Fig. 9. During the initial moments of the simulation, the rotor remains motionless while the stator becomes energized. After $15 \mathrm{ms,} \mathrm{rotation} \mathrm{begins}$ at the top of the torque amplitude sinusoid. The transient period ends at $0.13 \mathrm{~s}$, which is followed by constant operation at around $500 \mathrm{~N} \cdot \mathrm{m}$. This results in a power of $314 \mathrm{~kW}$ with an approximate power density above 18 $\mathrm{kW} / \mathrm{kg}$, which does not consider anything other than the motor's electromagnetic active components. The slow trend shown in Fig. 9 after the transient is caused by the effect of the currents settling in the rotor stacks.

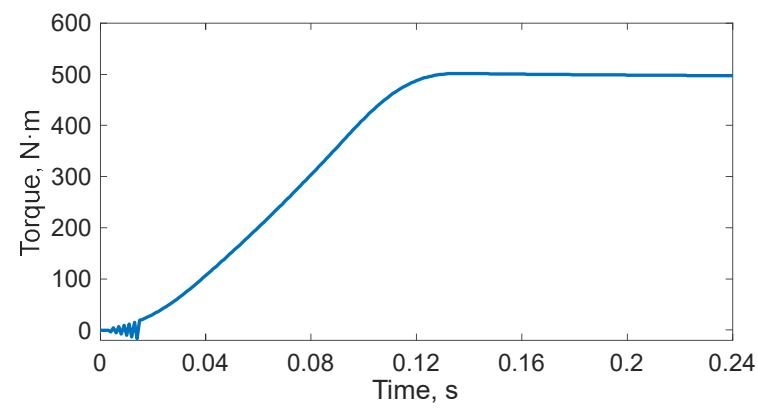

Fig. 9 Torque level yield during the motoring simulation.

\section{Stack losses}

The electromagnetic losses in each stack are computed from the 2D model using the numeric solution simply as the Joule heating in the superconducting region times the active length of the machine $L_{M}[23]$ : 


$$
P_{\text {loss }}=L_{M} \int_{\text {stack }} \boldsymbol{E} \cdot \boldsymbol{J} d S
$$

The average result of applying Eq. (6) to the 20 stacks is shown in Fig. 10. It is noticeable that the electromagnetic transient produced by ramping the stator currents sharply increases the losses in the stack by more than five times those expected under steady state. This occurs under a rather severe evolution: from zero to full torque in $0.13 \mathrm{~s}$. This behavior could be an important consideration if the motor is expected to be used for steering or attitude control, under continuous and rapid variations in the commanded power. On the other hand, the total losses for the full rotor remains at manageable levels for the cooling system at below $100 \mathrm{~W}$ in all cases and much less $(\sim 13 \mathrm{~W})$ while at steady state [15].

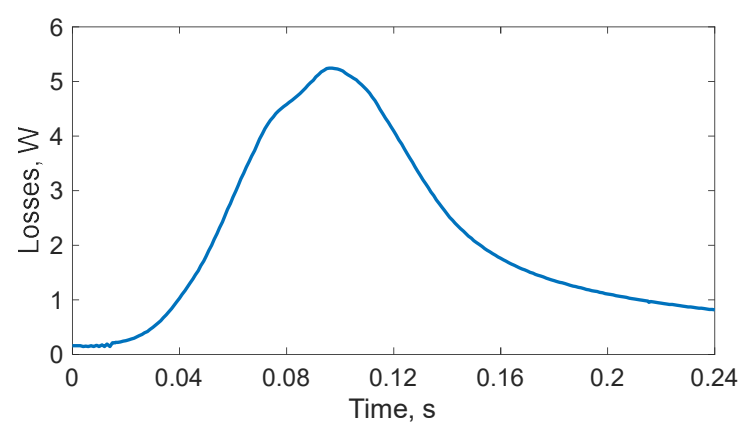

Fig. 10 Losses in a stack during the motoring simulation.

\section{Demagnetization}

The macroscopic nature of the currents circulating in the stacks indicates that any variations in the magnetic flux density will influence them. Cross-fields stir the current vortexes, which breaks their pattern and effectively demagnetizes the superconductor [11]. This effect is more sensitive to the amplitude of the cross-field and only proportional to its frequency (constant for the same number of cycles). Therefore, it is expected that demagnetization increases more with a rising torque rather than with the speed of the machine [24].

Figure 11 shows the results of laboratory tests that used an actual rotating electric machine with a 9-layer insulated AMSC stack $(<1 \mathrm{~mm})$ fixed to the rotor surface, which is magnetized and rotated in a liquid nitrogen environment $(77 \mathrm{~K})$ at $10 \mathrm{~Hz}$. As the cross-field (estimated at $25 \mathrm{mT}$ ) is applied from $t=20 \mathrm{~s}$ onwards, the peak back-electromotive (back-emf) force measured in the stator (for $1 \mathrm{~V}$ corresponds roughly to $100 \mathrm{mT}$ trapped in the stack) portrays a two-stage evolution: initial sharp decrease followed by a slow decay [25]. While more than $40 \%$ of the initial magnetization is rapidly lost despite the relatively small cross-field, the remaining $60 \%$ lasts for several minutes. 
The cross-field levels obtained from the numerical simulations using the combined formulation decrease the values obtained using the conventional model from Table 2 due to the saturation of the poles. The amplitude computed is around $10 \mathrm{mT}$ for the side of the stack facing the airgap (for a trapped field of $0.64 \mathrm{~T}$ ) during steady state conditions at full load. Therefore, some demagnetization is expected to occur in the motor, especially during the transient periods. However, there should be sufficient flux to perform the planned demonstrator tests, provided the evolution of this phenomenon is similar to that measured in Fig. 11.

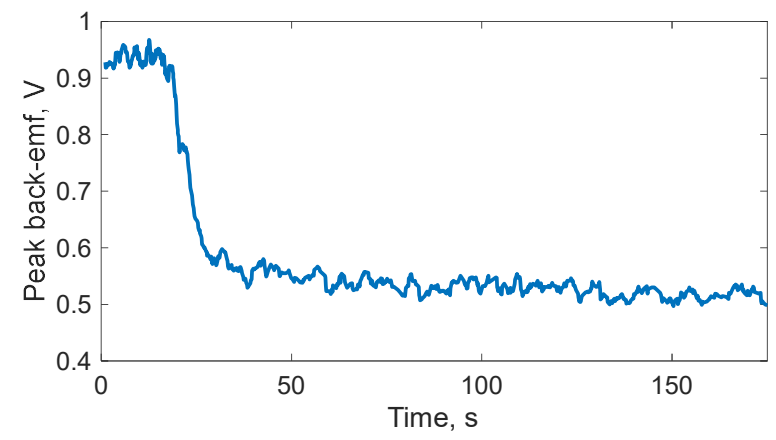

Fig. 11 Peak back-emf induced from a rotating stack as a cross-field is applied.

\section{Conclusions}

This work presents the results of simulating a novel rotor architecture that was specifically developed to use stacks of superconductive tape as magnetic flux sources. A new computational approach allows modeling the behavior of superconducting regions during the operation of a rotating electric machine. The procedure permits initializing the state of the machine under realistic conditions and to obtain its performance characteristics, such as its torque, electromagnetic losses in the stacks, and some insights into their demagnetization rate. According to these results, a fully superconducting motor based on stacks is feasible, although several problems persist, such as a modest trapped flux, flux leakage around the stacks, and some level of demagnetization. Nevertheless, the novel rotor lay-out reduces their influence and provides room for improvement. The task of confirming these results with experimental tests once the demonstrator becomes operational remains.

\section{Funding Sources}

This research is financially supported by the European Union's Horizon 2020 research innovation programme under grant agreement No 7231119 (ASuMED consortium) and EPSRC grant EP/P000738/1. 


\section{Acknowledgments}

The authors would like to express their gratitude to P. R. Librizzi for correcting the draft manuscript.

\section{References}

[1] Felder, J. L., Kim, H. D., and Brown, G. V., “Turboelectric Distributed Propulsion Engine Cycle Analysis for HybridWing-Body Aircraft," 47th AIAA Aerospace Sciences Meeting including The New Horizons Forum and Aerospace Exposition, Orlando, Florida, 2009, pp. 2009-1132. doi: $10.2514 / 6.2009-1132$

[2] Felder, J. L., Kim, H. D., Brown, G. V., and Chu, J., “An Examination of the Effect of Boundary Layer Ingestion on Turboelectric Distributed Propulsion Systems," 49th AIAA Aerospace Sciences Meeting including The New Horizons Forum and Aerospace Exposition, Orlando, Florida, 2011, pp. 2011-300.

doi: $10.2514 / 6.2011-300$

[3] Patel, A., Climente-Alarcon, V., Baskys, A., Glowacki, B. A., and Reis, T., "Design Considerations for Fully Superconducting Synchronous Motors Aimed at Future Electric Aircraft," IEEE 5th International Conference on Electrical Systems for Aircraft, Railroad, Ship Propulsion and Road Vehicles and International Transportation Electrification Conference, Nottingham, UK, 2018, pp. 1-6.

doi: 10.1109/ESARS-ITEC.2018.8607734

[4] Pyrhönen, J., Jokinen, T., and Hrabovcová, V., Design of Rotating Electrical Machines, $2^{\text {nd }}$ ed., Wiley, New York, USA, 2014.

[5] Song, X., Bührer, C. et al., "Designing and basic experimental validation of the world's first MW-class direct-drive superconducting wind turbine generator," IEEE Transactions on Energy Conversion, (accepted for publication) doi: 10.1109/TEC.2019.2927307

[6] Epstein, A. H. and O’Flarity, S. M., "Considerations for Reducing Aviation's CO2 with Aircraft Electric Propulsion," AIAA Journal of Propulsion and Power, Vol. 35, No. 3, 2019, pp. 572-582

doi: $10.2514 / 1 . B 37015$

[7] Kalsi, S. S., Weeber, K., Takesue, H., Lewis, C., Neumueller, H.-W., and Blaugher, R. D., "Development Status of Rotating Machines Employing Superconducting Field Windings," Proceedings IEEE, Vol. 92, No. 10, 2004, pp. 16881704.

doi: 10.1109/JPROC.2004.833676

[8] Shengnan Zou, "Magnetization of High Temperature Superconducting Trapped-Field Magnets," Ph.D. Dissertation, KIT Scientific Publishing, Karlsruhe, $\quad$ Germany, $2017 . \quad$ (Available on-line: https://publikationen.bibliothek.kit.edu/1000073152). doi:10.5445/IR/1000073152 
[9] Patel, A., Baskys, A., Mitchell-Williams, T., McCaul, A., Coniglio, W., Hänisch, J., Lao, M., and Glowacki, B. A., “A Trapped Field of $17.7 \mathrm{~T}$ in a Stack of High Temperature Superconducting Tape," Superconductor Science and Technology, Vol. 31 No. 9, 2018, 09LT01.

doi: 10.1088/1361-6668/aad34c

[10] Iwasa, Y., Case Studies in Superconducting Magnets, Design and Operational Issues, $2^{\text {nd }}$ ed., Springer, New York, USA, 2009.

[11] Campbell, A., Baghdadi, M., Patel, A., Zhou, D., Huang, K. Y., Shi, Y., and Coombs, T., "Demagnetisation by Crossed Fields in Superconductors," Superconductor Science and Technology, Vol. 30, No. 3, 2017, 034005. doi: $10.1088 / 1361-6668 / \mathrm{aa} 52 \mathrm{f} 2$

[12] Ahn, J., Han, C., Kim, C., and Choi, J., "Rotor Design of High-Speed Permanent Magnet Synchronous Motors Considering Rotor Magnet and Sleeve Materials," IEEE Transactions on Applied Superconductivity, Vol. 28 No. 3 , $2018,5201504$.

doi: 10.1109/TASC.2017.2786690

[13] Rao, J., Ronghai, Q., Jimin, M., and Xu, W., "Investigate the Influence of Magnetic Bridge Design on Mechanical Strength and Electromagnetic Characteristics in High Speed IPM Machines," 17th International Conference on Electrical Machines and Systems (ICEMS), Hangzhou, China, 22-25 Oct. 2014, pp. 22-27. doi: 10.1109/ICEMS.2014.7013444

[14] Brambilla, R., Grilli, F., Martini, L., Bocchi, M., and Angeli, G., “A Finite-Element Method Framework for Modelling Rotating Machines with Superconducting Windings," IEEE Transactions on Applied Superconductivity, Vol. 28, No. 5, $2018,5207511$. doi: 10.1109/TASC.2018.2812884

[15] Perez, A., van der Woude, R. R., and Dekker, R., "Rotor Cooling Concept for the ASuMED Superconductive Motor," IOP Conference Series: Materials Science and Engineering, Vol. 502, No. 1, 2019, 012139. doi:10.1088/1757-899X/502/1/012139

[16] Climente-Alarcon, V., Patel, A., Baskys, A., and Glowacki, B. A., "Design Considerations for Electric Motors Using Stacks of High Temperature Superconducting Tape as Permanent Magnets", IOP Conference Series: Materials Science and Engineering, Vol. 502, No. 1, 2019, 012182. doi:10.1088/1757-899X/502/1/012182

[17] Zhang, M., “A New World Record for Superconducting Trapped Field Magnet,” Superconductor Science and Technology, 2019. (in press, https://doi.org/10.1088/1361-6668/ab17e)

[18] Patel A., Usoskin A., Baskys A., Hopkins S. C., and Glowacki B. A., "Trapped field profiles for $40 \mathrm{~mm}$ wide superconducting tape pieces,” Journal of Superconductivity and Novel Magnetism, Vol. 28, No. 2, 2015, pp. 397-401 doi: $10.1007 / \mathrm{s} 10948-014-2665-2$ 
[19] Arkkio, A., "Analysis of Induction Motors Based on the Numerical Solution of the Magnetic Field and Circuit Equations,"

PhD. Dissertation, Aalto University, Finland, 1986 (on-line: http://lib.tkk.fi/Diss/198X/isbn951226076X/)

[20] Kim, Y. B., Hempstead, C. F., and Strnad, A. R., "Flux-flow Resistance in Type-II Superconductors," Physical Review, Vol. 139, No. 4A, 1965, pp. 1163-1172.

doi: 10.1103/PhysRev.139.A1163

[21] Climente Alarcon, V., Patel, A., Baskys, A., and Glowacki, B. A., "Computation of Superconducting Stacks Magnetization in an Electrical Machine," IEEE Transactions on Applied Superconductivity, Vol. 29, nº 8, 5204506, Dec. 2019.

doi: $10.1109 / \operatorname{tasc} .2019 .2923537$

[22] Freels, J. D., "Implementation of Oak Ridge National Laboratory Software Quality Assurance Requirements for COMSOL 3.4," The COMSOL Conference 2008 Boston, Boston, MA, USA, 2008, pp. 1-5.

OSTI Identifier: 963910

[23] Rhyner, J., "Magnetic Properties and AC-losses of Superconductors with Power Law Current-Voltage Characteristics," Physica C, Vol. 212, Jul. 1993, pp. 292-300. doi: 10.1016/0921-4534(93)90592-E

[24] Baskys A., Patel A., and Glowacki B. A., "Measurements of crossed-field demagnetization rate of trapped field magnets at high frequencies and below 77 K," Superconductor Science and Technology, Vol. 31, 2018, 065011.

doi: 10.1088/1361-6668/aabf32

[25] Smara, A., Mineev, N., Climente-Alarcon, V., Patel A., Baskys, A., Glowacki, B. A., and Reis, T., "Experimental Assessment of Rotor Superconducting Stack Demagnetization in $\mathrm{LN}_{2}$ Environment," Superconductor Science and Technology, 2019, Vol. 32, no 8, 085009, 2019.

doi: 10.1088/1361-6668/ab20bf 